\title{
Characterization of ETS gene aberrations in select histologic variants of prostate carcinoma
}

\author{
Bo Han ${ }^{1,2,7}$, Rohit Mehra ${ }^{1,2,3,7}$, Khalid Suleman ${ }^{1,2}$, Scott A Tomlins ${ }^{1,2}$, Lei Wang, \\ Nishi Singhal ${ }^{1}$, Katherine A Linetzky ${ }^{1}$, Nallasivam Palanisamy ${ }^{1,2}$, Ming Zhou ${ }^{4}$, \\ Arul M Chinnaiyan ${ }^{1,2,3,5,6,8}$ and Rajal B Shah" ${ }^{1,2,3,6,8}$
}

${ }^{1}$ Michigan Center for Translational Pathology, University of Michigan Medical School, Ann Arbor, MI, USA; ${ }^{2}$ Department of Pathology, University of Michigan Medical School, Ann Arbor, MI, USA; ${ }^{3}$ Comprehensive Cancer Center, University of Michigan Medical School, Ann Arbor, MI, USA; ${ }^{4}$ Department of Anatomic Pathology, Cleveland Clinic, Cleveland, OH, USA; ${ }^{5}$ Howard Hughes Medical Institute, University of Michigan Medical School, Ann Arbor, MI, USA and ${ }^{6}$ Department of Urology, University of Michigan Medical School, Ann Arbor, MI, USA

\begin{abstract}
Histologic variants of prostate carcinoma account for $5-10 \%$ of the disease and are typically seen in association with conventional acinar carcinoma. These variants often differ from the latter in clinical, immunophenotypic, and biologic potential. Recently, recurrent gene fusions between the androgen-regulated gene TMPRSS2 and the ETS transcription factors ERG, ETV1, ETV4, or ETV5 have been identified in a majority of conventional prostate carcinomas. However, the frequency and significance of this critical molecular event is unknown in the histologic variants of prostate carcinoma. Here, we used break-apart fluorescence in situ hybridization to assess TMPRSS2 and ETS aberrations in a series of select histologic variants: foamy gland carcinoma $(N=17)$, ductal adenocarcinoma $(N=18)$, mucinous carcinoma $(N=18)$, and small cell carcinoma $(N=7)$. A histologic variation of acinar adenocarcinoma, demonstrating glomeruloid morphology $(N=9)$, was also investigated. Overall, $55 \%$ of histologic variant or variation morphologies demonstrated ETS aberrations (ERG in $54 \%$ and ETV1 in 1\%). TMPRSS2:ERG fusion was identified in 83\% (15/18), 71\% (5/7), 50\% (9/18), 33\% (3/9), and 29\% (5/17) of mucinous, small cell, ductal, glomeruloid, and foamy gland prostate carcinomas, respectively. Previously, we reported that $100 \%$ of androgen-independent metastatic prostate carcinomas harboring TMPRSS2:ERG gene fusion were associated with interstitial deletion (Edel). Interestingly, ERG rearrangement in small cell carcinomas occurred exclusively through Edel, supporting the notion that TMPRSS2:ERG with Edel is an aggressive molecular subtype. SPINK1, a biomarker expressed exclusively in a subset of ETS negative prostate carcinomas, was expressed in $6 \%$ of ETS negative histologic variants, specifically in ductal adenocarcinoma. Notably, 88\% (43/49) variant morphologies in this cohort showed concordance of TMPRSS2:ERG fusion with associated conventional acinar type, suggesting that variant morphology is clonally related to the latter. Overall, our data provide insight into the origin, molecular mechanism, and phenotypic association of ETS fusions in histologic variants of prostate carcinoma.

Modern Pathology (2009) 22, 1176-1185; doi:10.1038/modpathol.2009.79; published online 22 May 2009
\end{abstract}

Keywords: ETS; rearrangement; histologic variant; prostate carcinoma; fluorescence in situ hybridization

It is estimated that more than 186320 new cases of prostate carcinoma will be diagnosed in the United States in 2008, with approximately $90 \%$ of those

Correspondence: Dr RB Shah, MD, Department of Pathology and Urology, University of Michigan Medical School, 2G332 UH, 1500 East Medical Center Drive, Ann Arbor, MI 48109, USA.

E-mail: rajshah@umich.edu

${ }^{7}$ These authors contributed equally to this work.

${ }^{8}$ These authors share senior authorship.

Received 9 February 2009; revised and accepted 27 April 2009; published online 22 May 2009 cases being classified as conventional acinar type. ${ }^{1}$ Various histologic variants of prostate carcinoma, such as mucinous, ductal, foamy gland, and small cell neuroendocrine carcinoma, contribute to $5-10 \%$ of the disease. ${ }^{2,3}$ These variants are typically seen in association with conventional prostate carcinoma, and often differ from latter in clinical, immunophenotypic, genetic, and biologic potential. ${ }^{2,3}$ For example, small cell carcinoma and ductal adenocarcinoma are known to have a distinctly aggressive clinical behavior and poor prognosis. ${ }^{4}$ 
However, it is unclear whether these histologic variants are genetically distinct from the conventional acinar type.

We recently identified the fusion of the 5 -untranslated region of TMPRSS2 (21q22.3) with the ETS family members ERG (21q22.2), ETV1 (7q21.2), ETV4 (17q21), and ETV5 (3q27.2) in a majority of conventional acinar prostate carcinomas. ${ }^{5-8}$ We and others also identified novel $5^{\prime}$ partner genes of ETV1, ETV4, and ETV5 in prostate carcinoma, including SLC45A3, HERV-K_22q11.23, C15ORF21, HNRPA2B1, FLJ35294, CANT1, $K L K 2$, and $D D X 5 .{ }^{8-10}$ Among these aberrations, TMPRSS2:ERG fusion is the most prevalent, occurring in $\sim 50-70 \%$ of localized carcinomas and $\sim 40 \%$ of androgen-independent metastatic carcinomas. ${ }^{11-15}$ As TMPRSS2 and ERG are located $\sim 3 \mathrm{Mb}$ apart on chromosome 21, the rearrangement between them occurs either through translocation or by an interstitial deletion (Edel). ${ }^{15}$ Emerging data have suggested association of TMPRSS2:ERG fusion, specifically associated with Edel, resulting in a more aggressive phenotype in clinically localized as well as in androgen-independent metastatic prostate carcinoma. ${ }^{12,14-18}$ Of note, multiple studies have indicated that ETS fusion-positive and ETS fusionnegative carcinomas have distinct transcriptional signatures across profiling studies. ${ }^{19,20}$ Recently, we have identified SPINK1 overexpression exclusively in a subset of ETS fusion-negative prostate carcinomas. ${ }^{21}$ The ETS fusion-positive cases most likely define a distinct class of prostate carcinoma with potential implications for early diagnosis, prognosis, and rational therapeutic targeting.

Similar to BCR:ABL1 fusion leukemias ${ }^{22}$ microsatellite unstable colon carcinomas $^{23}$ or breast carcinomas with BRCA mutations, ${ }^{24}$ ETS gene fusions in prostate carcinoma have been reported to be associated with certain morphologic features, which predict underlying genetic association. Mosquera et $a l^{25}$ identified blue-tinged mucin, cribriform growth pattern, macronucleoli, intraductal tumor spread, and signet-ring cell features to be significantly associated with TMPRSS2:ERG fusion status. Tu et $a l^{13}$ also observed that mucinpositive prostate carcinomas more often harbor
TMPRSS2:ERG gene fusions when compared to mucin-negative tumors. These findings suggest a potential contributory function of ETS aberrations in development of these specific morphologic subtypes. However, the frequency, molecular subtypes, and clonality of TMPRSS2:ETS gene aberrations in histologic variants of prostate carcinoma in relation to conventional acinar type are currently unknown. In this study, we comprehensively assessed genomic aberrations of ETS (ERG, ETV1, ETV4, and ETV5) transcription factors and their known $5^{\prime}$ fusion partner, TMPRSS2, by fluorescence in situ hybridization (FISH) assay in a series of prostate carcinoma cases of histologic variants.

\section{Materials and methods}

\section{Study Population, Clinical Data, and Case Selection}

Drawing from a sample set of over 400 radical prostatectomy resections and transurethral resections of the prostate performed between 2004 and 2006 and 56 rapid autopsies of men that died of androgen-independent metastatic prostate carcinoma, 69 cases of select histologic variants or variation of prostate carcinomas were identified for the study. None of the patients who underwent radical prostatectomy received preoperative radiation or androgen deprivation therapy. The variant prostate carcinoma spectrum included 18 mucinous carcinomas, 17 foamy gland carcinomas, 18 ductal adenocarcinomas, and 7 small cell carcinomas. Signet-ring cell and sarcomatoid variants were not included mainly due to their extreme rarity in surgical pathology practice. Of note, nine prostate carcinoma cases with prominent glomeruloid morphology were also investigated as a histologic variation of acinar adenocarcinoma. Overall, only those cases where the variant histologic components made up over $25 \%$ of the tumor volume were included. All ductal adenocarcinoma cases demonstrated ductal component $\geq 65 \%$ of the tumor volume. Patient demographics for each type are shown in Table 1 and all cases were obtained from pathology archives of University of Michigan Hospital and Cleveland Clinic. To better understand the

Table 1 Clinical and pathologic demographics of patients with prostate carcinoma with histologic variants or variations

\begin{tabular}{|c|c|c|c|c|c|c|c|c|c|c|c|}
\hline \multirow[b]{2}{*}{ Histology variant } & \multicolumn{2}{|c|}{ Age (years) } & \multicolumn{2}{|c|}{ Tumor size $(\mathrm{cm})$} & \multicolumn{2}{|c|}{ Pathology stage } & \multicolumn{3}{|c|}{ Preoperative PSA (ng/ml) } & \multicolumn{2}{|c|}{ PSA recurrence } \\
\hline & $\leq 60$ & $>60$ & $<1$ & $\geq 1$ & $\leq T 2 b$ & $\geq T 2 C$ & $\leq 4$ & $4-7$ & $>7$ & No & Yes \\
\hline Mucinous & 10 & 8 & 5 & 13 & 7 & 0 & 1 & 2 & 4 & 4 & 1 \\
\hline Ductal & 17 & 2 & 2 & 17 & 9 & 2 & 0 & 6 & 5 & 5 & 5 \\
\hline Foamy & 12 & 5 & 2 & 15 & 13 & 1 & 0 & 8 & 6 & 12 & 1 \\
\hline Glomeruloid & 4 & 4 & 2 & 6 & 5 & 0 & 0 & 4 & 1 & 4 & 0 \\
\hline All variants ${ }^{\mathrm{a}}$ & 43 & 19 & 11 & 51 & 34 & 3 & 1 & 20 & 16 & 25 & 7 \\
\hline
\end{tabular}

${ }^{a}$ Small cell carcinoma represented in this cohort include six patients with metastatic prostate carcinoma and one patient with transurethral resection of the prostate. Clincal information is not available for these cases. 
clonal relation between paired histologic variant and conventional acinar carcinoma, we determined whether the prostate carcinoma was focal or multifocal as previously described. ${ }^{26}$ Briefly, tumor maps were generated by tracking each section and reconstructing them as a whole-mount section. A carcinoma was considered multifocal if it was $3 \mathrm{~mm}$ or more from the closest carcinoma in any single section, or if it was $4 \mathrm{~mm}$ or more from the closest carcinoma on the adjacent section above or below. In the instance of non-multifocal prostate carcinoma, representative tumor blocks were selected that contained both variant morphology and conventional acinar prostate carcinoma. For multifocal cases, variant morphology and conventional acinar carcinoma were represented from the same focus if available. However, when that was not possible, independent tumor foci were represented.

\section{Tissue Microarray Construction}

Three cores $(0.6 \mathrm{~mm}$ in diameter) were taken from each area of interest representing variant morphology and, when possible, paired conventional prostate carcinoma. Morphologic diagnosis was confirmed on H\&E-stained sections of tissue microarray before FISH assessment. The detailed clinical, pathologic, and tissue microarray data were maintained on a secure relational database as previously described. ${ }^{11}$ This study was approved by the institutional review board at the University of Michigan Medical School and Cleveland Clinic. Radical prostatectomy series at the University of Michigan Hospital and the Rapid Autopsy Program are part of the University of Michigan Prostate Cancer Specialized Program of Research Excellence Tissue Core (SPORE).

\section{FISH and Assessment of TMPRSS2:ETS Fusion}

Interphase FISH was performed as previously described. ${ }^{9,11}$ Bacterial artificial chromosomes (BACs) were obtained from the BACPAC Resource Center (Oakland, CA, USA), and probes were prepared as described. ${ }^{5}$ The integrity and correct localization of all probes was verified by hybridization to metaphase spreads of normal peripheral lymphocytes. For detection of TMPRSS2 and ETS rearrangements, we used the following BAC clones as probes: RP11-35C4 ( $5^{\prime}$ to TMPRSS2) and RP11120C17 ( $3^{\prime}$ to TMPRSS2), RP11-95I21 ( $5^{\prime}$ to $E R G$ ) and RP11-476D17 ( $3^{\prime}$ to ERG), RP11-703A4 (5' to ETV1) and RP11-124L22 ( $3^{\prime}$ to ETV1), RP11-436J4 ( $5^{\prime}$ to ETV4) and RP11-100E5 ( $3^{\prime}$ to ETV4), and RP11$379 \mathrm{C} 23$ ( $5^{\prime}$ to ETV5) and RP11-1144N13 ( $3^{\prime}$ to ETV5). Slides were examined using an Imaging Zeiss microscope (Carl Zeiss, Oberkochen, Germany) equipped with ISIS image processing software (MetaSystems, USA). FISH signals were scored manually ( $\times 100$ oil immersion objective) in mor- phologically intact and nonoverlapping nuclei by two pathologists (BH and RM), and a minimum of 50 carcinoma cells from each site were recorded. Carcinoma sites with very weak or no signals were recorded as insufficiently hybridized. Cases lacking tumor tissue in all three cores were excluded.

A previously validated break-apart probe FISH approach was used to investigate gene fusion involving TMPRSS2 and ETS transcription factors (ERG, ETV1, ETV4, and ETV5). ${ }^{11}$ Briefly, normal signal patterns for TMPRSS2 and ETS family genes were indicated by two pairs of colocalized green and red signals (Figure 1a3); a translocation was indicated by break-apart of one of the two colocalized signals (Figure 1c3); a deletion was indicated by the loss of either one $5^{\prime}$ or $3^{\prime}$ signal (Figure 1b3); a duplication was indicated as the presence of two or more $5^{\prime}$ or $3^{\prime}$ signals (Figure 1e3).

\section{Immunohistochemistry}

Immunohistochemistry for SPINK1 antibody was performed as previously described. ${ }^{21,27}$ Briefly, a mouse monoclonal antibody against SPINK1 (H00006690-M01; Abnova, Taipei City, Taiwan) was applied on tissue microarray using 1:1000 dilution, and incubated overnight at $4^{\circ} \mathrm{C}$ following standard LSAB immunohistochemical staining protocol. ${ }^{21}$ Cases presenting cytoplasmic staining in any cancerous epithelial cells were deemed positive.

\section{Results}

\section{Frequency of TMPRSS2:ETS Fusions}

Our FISH break-apart probe strategy revealed ETS aberrations in $55 \%(38 / 69)$ of prostate carcinoma cases with histologic variants and glomeruloid histologic variation. Overall, 54 and $1 \%$ of the cases were rearranged for $E R G$ and $E T V 1$, respectively. No case with either ETV4 or ETV5 rearrangement was identified in this cohort. As shown in Table 2, ERG rearrangement was identified in $83 \%(15 / 18)$ of mucinous carcinomas and $71 \%(5 / 7)$ of small cell carcinomas, followed by ductal adenocarcinomas and prostate carcinoma cases with glomeruloid morphology in 50\% (9/18) and 33\% (3/9), respectively. By contrast, only 29\% (5/17) of cases showed $E R G$ rearrangement in foamy gland carcinoma. Overall, $100 \%$ of the prostate carcinoma cases with ERG rearrangement harbored TMPRSS2 as the $5^{\prime}$ fusion partner. Among these, 46\% (17/37) were fused through deletion of its $5^{\prime}$ end to TMPRSS2, which is comparable to previous reports in conventional acinar carcinomas. ${ }^{9,11}$ ETV1 rearrangement was observed in only one prostate carcinoma case with glomeruloid morphology in this cohort. However, no rearrangement for the known $5^{\prime}$ fusion partners (TMPRSS2, SLC45A3, HNRPA2B1, HERVK_22q11.23, C15ORF21) was identified in this case 

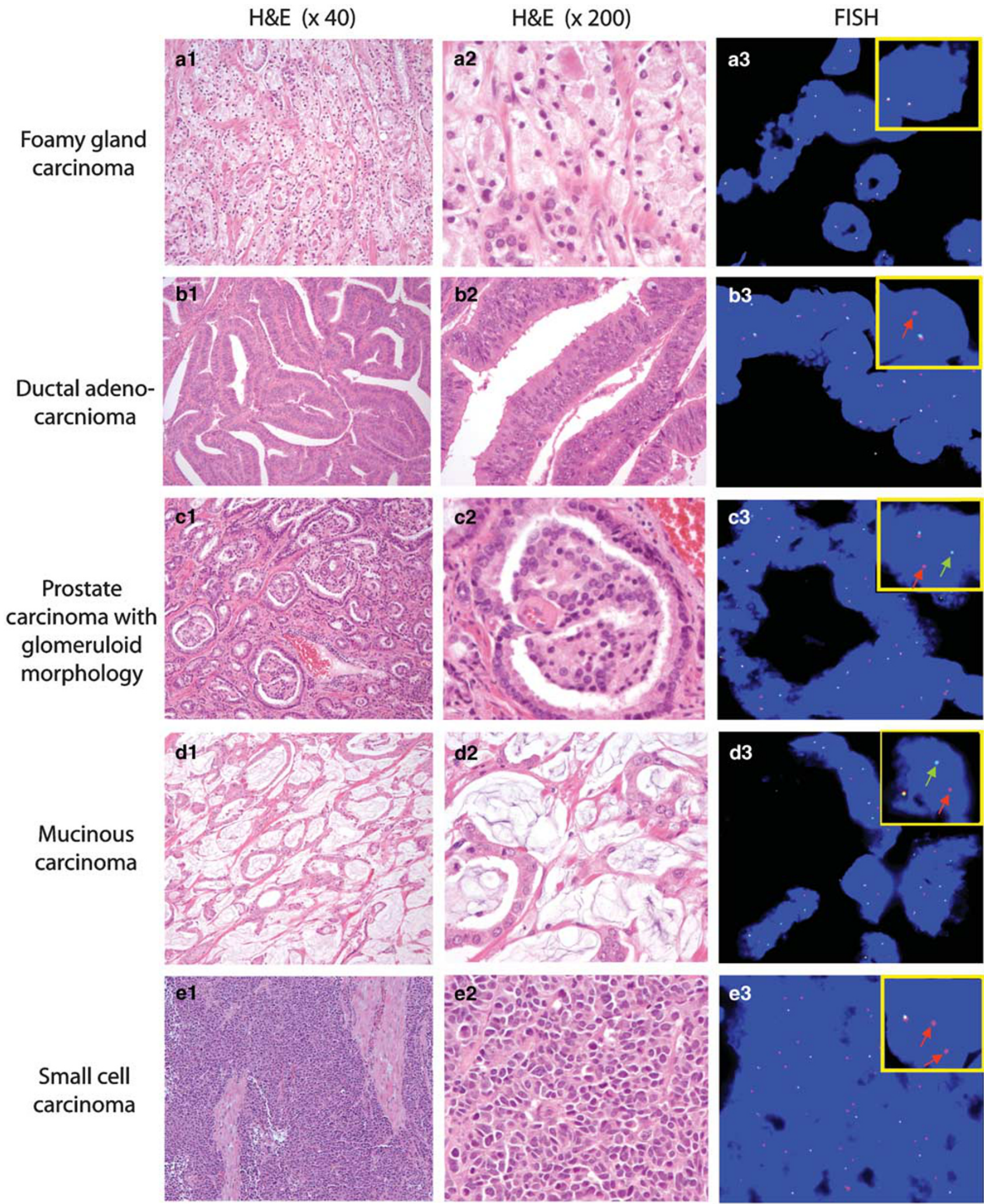

Figure 1 H\&E staining and corresponding FISH images of ERG rearrangement in different histologic variants and glomeruloid histologic variation of acinar adenocarcinoma. Representative morphologic images of four histologic variants (foamy gland, mucinous, ductal, and small cell) as well as prostate carcinoma with glomerulation features were shown at lower magnification (a1-e1, left row) and high magnification (a2-e2, middle row). ERG break-apart FISH assay was performed and corresponding images were shown at right row (a3-e3). The rectangular boxes show magnified images illustrating the $E R G$ rearrangement pattern. $E R G$ rearrangement negative case was indicated by two pairs of colocalized green and red signals. ERG rearrangement positive (with deletion) case showed loss of one greenlabeled probe $5^{\prime}$ to ERG. ERG rearrangement positive (translocation) case showed one pair of split 5' (green) and 3' (red) signals. 
Table 2 Summary of ETS aberrations in histologic variants and variations of prostate carcinoma

\begin{tabular}{|c|c|c|c|c|c|c|}
\hline \multirow[b]{2}{*}{ Histologic variants/variations } & \multicolumn{3}{|c|}{ ERG } & \multirow[b]{2}{*}{ ETV1 } & \multirow[b]{2}{*}{ ETV4 } & \multirow[b]{2}{*}{ ETV5 } \\
\hline & Rearranged & $\begin{array}{c}\text { Fusion through } \\
\text { deletion }\end{array}$ & $\begin{array}{l}\text { Fusion through } \\
\text { translocation }\end{array}$ & & & \\
\hline Foamy gland & $29 \%(5 / 17)$ & $20 \%(1 / 5)$ & $80 \%(4 / 5)$ & $0 \%(0 / 16)$ & $0 \%(0 / 14)$ & $0 \%(0 / 17)$ \\
\hline Large duct & $50 \%(9 / 18)$ & $56 \%(5 / 9)$ & $44 \%(4 / 9)$ & $0 \%(0 / 18)$ & $0 \%(0 / 17)$ & $0 \%(0 / 18)$ \\
\hline Glomeruloid & $33 \%(3 / 9)$ & $33 \%(1 / 3)$ & $67 \%(2 / 3)$ & $14 \%(1 / 7)$ & $0 \%(0 / 8)$ & $0 \%(0 / 9)$ \\
\hline Mucinous & $83 \%(15 / 18)$ & $33 \%(5 / 15)$ & $67 \%(10 / 15)$ & $0 \%(0 / 18)$ & $0 \%(0 / 18)$ & $0 \%(0 / 18)$ \\
\hline Small cell & $71 \%(5 / 7)$ & $100 \%(5 / 5)$ & $0 \%(0 / 5)$ & $0 \%(0 / 7)$ & $0 \%(0 / 7)$ & $0 \%(0 / 7)$ \\
\hline
\end{tabular}

(data not shown). Representative morphology and corresponding FISH images of each histologic variant morphology and glomeruloid histologic variation of acinar adenocarcinoma are shown in Figure 1.

\section{Duplication of TMPRSS2:ERG Fusion with Edel in Small Cell Carcinoma}

Small cell carcinoma of the prostate is an extremely rare but highly aggressive variant with poor prognosis. ${ }^{4}$ In this cohort, $71 \%(5 / 7)$ of small cell carcinomas harbored TMPRSS2:ERG fusions. Of note, all five of these TMPRSS2:ERG fusion cases were from the University of Michigan androgenindependent metastatic prostate carcinoma autopsy cohort. ${ }^{12}$ Interestingly, TMPRSS2:ERG fusions through Edel were exclusively identified in all five of these cases. In addition, as shown in Figure 1e1-3, the break-apart probe FISH assay revealed that four out of five cases demonstrated two copies of the $3^{\prime}-E R G$ signals as well as two copies of the $5^{\prime}$-TMPRSS 2 signal, suggesting the duplication of TMPRSS2:ERG fusions. No genetic aberrations involving ETV1, ETV4, or ETV5 were identified in small cell carcinoma variant.

\section{Clonal Nature of TMPRSS2:ERG Fusion in Histologic Variants and Glomeruloid Histologic Variation of Acinar Adenocarcinoma}

A total of 98 tumor foci from 49 cases were interrogated for clonality of TMPRSS2:ETS aberrations. We evaluated paired histologic variant/variation vs conventional acinar tumor foci from each case, 27 out of 49 cases (55\%) showed ERG aberrations at least at one tumor focus, whereas the remaining $22(45 \%)$ cases lacked $E R G$ aberrations in all tumor foci (Figure 2). Overall, 43 out of 49 cases $(88 \%)$ were concordant for TMPRSS2:ERG fusion or concordant, by lack of ETS rearrangement, in any tumor foci. Figure 3 represents a reconstructed map of the prostatectomy sections in a patient with ductal adenocarcinoma variant. By contrast, discordance of TMPRSS2:ERG status was observed in six cases. Upon reviewing histologic specimens, paired histologic variant and conventional acinar tumor foci were independent of each other in these cases and represent multifocal prostate carcinoma. Therefore, these data suggest that the in vast majority of patients with prostate carcinoma, histologic variants are clonally related to associated conventional acinar prostate carcinoma.

\section{SPINK1 Immunoexpression in Histologic Variants and Glomeruloid Histologic Variation of Acinar} Adenocarcinoma

In the current study, $45 \%$ of cases did not harbor any ETS aberrations. This prevalence is comparable with that reported previously in conventional acinar prostate carcinoma. ${ }^{9,11}$ Previously, using a Cancer Outlier Profile Analysis (COPA) strategy, we identified SPINK1 outlier expression exclusively in ETS rearrangement negative acinar carcinomas $(\sim 11 \%$ of total cases). ${ }^{21}$ To characterize SPINK1 expression in the histologic variants, we performed immunohistochemistry of SPINK1 on the tissue microarray. Overall, SPINK1 overexpression was identified in $6 \%(2 / 31)$ of ETS negative cases, both of which were from ductal adenocarcinoma variant and were TMRPSS2:ETS negative (Figure 4).

\section{Discussion}

To the best of our knowledge, this is the first study that comprehensively characterized TMPRSS2:ETS aberrations in the histologic variants of prostate carcinoma. On the basis of the break-apart probe strategy, we found that $55 \%$ of the variant morphologies in this cohort harbored carcinoma aberrations, most of which demonstrated TMPRSS2:ERG fusions. This frequency of gene fusions is comparable to that reported in clinically localized conventional acinar prostate carcinoma, ${ }^{9,11,13,14}$ which suggests that the high frequency of ETS aberrations present in localized acinar prostate carcinoma is also maintained in uncommon histologic variants. Of note, TMPRSS2:ERG fusion frequencies show a significant variation in different histologic variants of prostate carcinoma. A very high frequency of TMPRSS2:ERG fusion was found in mucinous 
T1 (uncommon histologic morphology)

T2 (Conventional prostate carcinoma morphology

\begin{tabular}{|c|c|c|c|c|c|c|c|c|c|c|c|c|}
\hline & Case No. & TMPRSS2 & ERG & ETV1 & ETV4 & ETV5 & TMPRSS2 & ERG & ETV1 & ETV4 & ETV5 & Multifocality \\
\hline \multirow{14}{*}{ 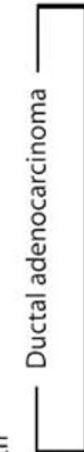 } & 1 & $3^{\prime}$ & $5^{\prime}$ & & & & $3^{\prime}$ & $5^{\prime}$ & & & & No \\
\hline & 2 & $3^{\prime}$ & $5^{\prime}$ & & & & $3^{\prime}$ & $5^{\prime}$ & & & & No \\
\hline & 3 & & & & & & & & & & & Yes \\
\hline & 5 & & $5^{\prime}$ & & & & $3^{\prime}$ & $5^{\prime}$ & & & & Yes \\
\hline & 6 & & & & & & & & & & & No \\
\hline & 7 & & & & & & & & & & & No \\
\hline & 8 & $3^{\prime}$ & $5^{\prime}$ & & & & $3^{\prime}$ & $5^{\prime}$ & & & & No \\
\hline & 9 & $3^{\prime}$ & $5^{\prime}$ & & & & 3 & $5^{\prime}$ & & & & No \\
\hline & 10 & & & & & & & & & & & No \\
\hline & 11 & & & & & & & & & & & Yes \\
\hline & 12 & & & & & & & & & & & Yes \\
\hline & 13 & & & & & & & & & & & No \\
\hline & 14 & & & & & & & & & & & Yes \\
\hline & 15 & & & & & & & & & & & Yes \\
\hline \multirow{6}{*}{ 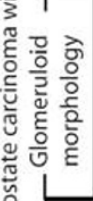 } & 16 & $3^{\prime}$ & $5^{\prime}$ & & & & 3 & $5^{\prime}$ & & & & Yes \\
\hline & 17 & & & & & & & & & & & Yes \\
\hline & 19 & & & & & & & & & & & No \\
\hline & 20 & & & & & & & & & & & Yes \\
\hline & 21 & & & & & & & & & & & No \\
\hline & 22 & & & & & & & & & & & Yes \\
\hline \multirow{13}{*}{$<$} & 23 & & & & & & & & & & & No \\
\hline & 24 & $3^{\prime}$ & $5^{\prime}$ & & & & $3^{\prime}$ & $5^{\prime}$ & & & & No \\
\hline & 25 & & & & & & & & & & & Yes \\
\hline & 26 & $3^{\prime}$ & $5^{\prime}$ & & & & & & & & & Yes \\
\hline & 27 & & & & & & & & & & & Yes \\
\hline & 28 & & & & & & & & & & & Yes \\
\hline & 29 & $3^{\prime}$ & $5^{\prime}$ & & & & $3^{\prime}$ & $5^{\prime}$ & & & & No \\
\hline & 30 & & & & & & & & & & & No \\
\hline & 31 & & & & & & & & & & & Yes \\
\hline & 32 & & & & & & & & & & & No \\
\hline & 33 & $3^{\prime}$ & $5^{\prime}$ & & & & $3^{\prime}$ & $5^{\prime}$ & & & & No \\
\hline & 34 & & & & & & & & & & & No \\
\hline & 35 & & & & & & & & & & & Yes \\
\hline \multirow{12}{*}{ 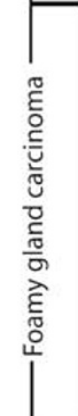 } & 38 & & & & & & & & & & & Yes \\
\hline & 39 & & & & & & & & & & & Yes \\
\hline & 40 & & & & & & & & & & & Yes \\
\hline & 41 & & & & & & & & & & & Yes \\
\hline & 42 & & & & & & & & & & & Yes \\
\hline & 43 & & & & & & & & & & & No \\
\hline & 44 & & & & & & & & & & & Yes \\
\hline & 45 & & & & & & & & & & & Yes \\
\hline & 46 & & & & & & & & & & & No \\
\hline & 47 & $3^{\prime}$ & $5^{\prime}$ & & & & $3^{\prime}$ & $5^{\prime}$ & & & & No \\
\hline & 48 & & & & & & & & & & & Yes \\
\hline & 49 & & & & & & & & & & & Yes \\
\hline
\end{tabular}

Translocation

5 ' deletion

3' deletion

Negative for rearrangement

Insufficient hybridization

Figure 2 Summary matrix of TMPRSS2 and ETS aberrations in different histologic variants, glomeruloid histologic variation of acinar adenocarcinoma, and associated conventional prostate carcinoma. Patients with prostate carcinoma of histologic variants or variation with associated conventional prostate carcinoma component were shown in the summary matrix. Patient case numbers are indicated on the left of the map. Each column represents one case; each row represents FISH evaluation for TMPRSS2 or ETS aberration at each tumor focus. TMPRSS2:ETS gene rearrangement status of cases with two tumor foci, histologic counterpart, and associated conventional prostate carcinoma were shown. Color legend signifies respective aberrations or availability.

carcinoma. Indeed, Mosquera et a ${ }^{25}$ have identified blue-tinged mucin as one of the morphologic features that was associated with TMPRSS2:ERG fusion. Further, they found a significant association between mucin-related genes (eg MUC1) expression and TMPRSS2:ERG fusion carcinoma. In addition, $\mathrm{Tu}$ et $a l^{13}$ found that mucin-positive prostate carcinomas more often harbored TMPRSS2:ERG gene fusions when compared to mucin-negative tumors. In line with these observations, our results, for the first time, suggest mucinous prostate carcinoma to be significantly associated with the TMPRSS2:ERG fusion. Although rare, linkage of special histologic subtypes to gene translocations in 


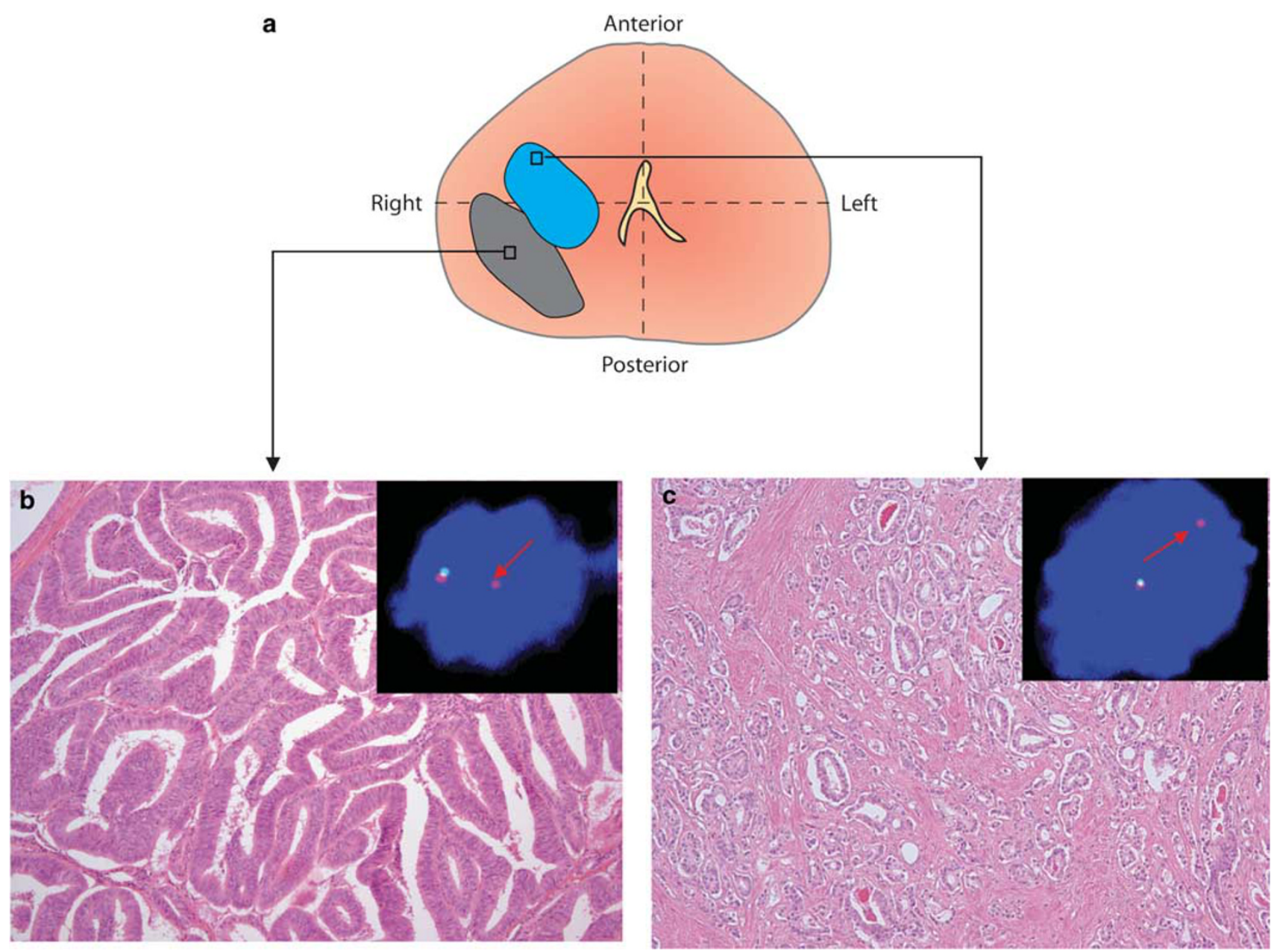

Figure 3 Representative example of ductal adenocarcinoma case. H\&E sections with two adjacent tumor foci of carcinoma showing concordant $E R G$ rearrangement. (a and b) Ductal adenocarcinoma component (gray). (a and c) Adjacent conventional acinar tumor foci (blue). (b and $\mathbf{c}$ inset) One yellow signal and individual red color indicating that fusion through deletion has occurred.

carcinomas has been documented. For example, Makretsov et $a 1^{28}$ have reported NTRK3:ETV6 fusion is restricted to the secretory breast carcinoma. It is unknown why mucinous carcinoma may be more likely to harbor TMPRSS2:ERG fusions, and this would be an interesting focus of future study. By contrast, a very low frequency of TMPRSS2:ERG fusion is observed in foamy gland carcinoma. Given distinct transcriptional signatures between ETS fusion-positive and ETS fusion-negative carcinomas across profiling studies, ${ }^{19}$ it is reasonable to speculate that the TMPRSS2:ERG fusion might alter molecular pathways favoring mucin secretion, instead of predisposing to a foamy gland phenotype.

Second, we systematically analyzed ETS aberrations in small cell carcinoma of prostate. Interestingly, we observed that TMPRSS2:ERG fusion in small cell carcinomas occurred exclusively through Edel. As TMPRSS2 and ERG are located $\sim 3 \mathrm{Mb}$ apart on chromosome 21, the rearrangement between them occurs either through translocation or by an Edel. ${ }^{15}$ Previously, in a FISH-based analysis of
445 prostate carcinoma cases, Attard et al ${ }^{16}$ correlated Edel gene fusions with poorer prognosis. Perner et $a l^{15}$ also observed a significant association of Edel gene fusions with high tumor stage and the presence of metastatic disease involving pelvic lymph node. Recently, we have reported that all androgen-independent metastatic prostate carcinomas in a warm autopsy series harboring TMPRSS2:ERG fusion were found to be associated with Edel. ${ }^{12}$ Taken together, correlating these data with our observations in this study, Edel potentially represents a molecular subtype that is correlated to higher tumor stage and recurrence, distinct histologic subtype with poor prognosis, evolution into an androgen-independent state, and eventually progression to metastasis. In addition, out of five fusion-positive small cell carcinomas, four cases demonstrated duplication of the TMPRSS2:ERG fusions. Attard et $a 1^{16}$ have reported that duplication of TMPRSS2:ERG fusion in combination with deletion of $5^{\prime}-E R G$ exhibited a very poor cause-specific survival. Recently, Mertz et $a l^{29}$ reported on 

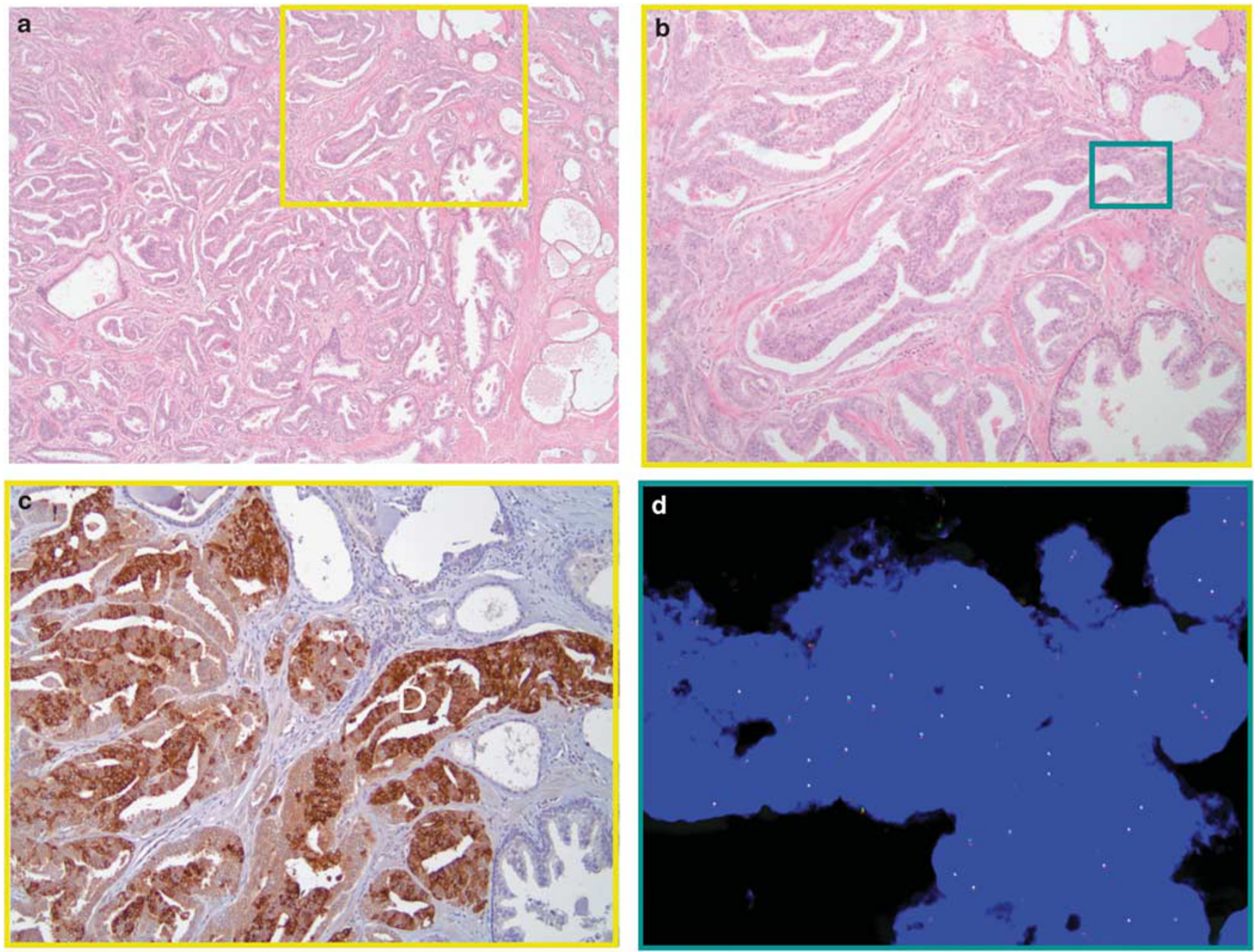

Figure 4 SPINK1 protein expression exclusively in ductal adenocarcinoma of prostate by immunohistochemistry. SPINK1 protein expression was evaluated in this cohort using immunohistochemistry. Histology of one ductal adenocarcinoma case is shown in a (H\&E, $\times 100)$ and in $\mathbf{b}$, yellow-boxed area in $\mathbf{a}(\mathrm{H} \& \mathrm{E}, \times 200)$, demonstrating complex branching and papillary cribriform structure. Strong cytoplasmic staining of SPINK1 was observed in ductal adenocarcinoma focus in c. (d) FISH image of the green-boxed area in b, displaying absence of $E R G$ rearrangement, indicated by two pairs of colocalized red and green signals.

androgen receptor negative NCI-H600 cell line, derived from metastatic site of a prostatic small cell carcinoma patient. They identified that NCI-H600 harbored the TMPRSS2:ERG fusion with a homozygous Edel, which was consistent with our findings. However, considering of limited number of small cell carcinomas in the current study, additional investigation with large cohort may further define ETS gene aberrations in small cell carcinoma of the prostate.

Histologic variants or histologic variation of prostate carcinoma are usually associated with conventional acinar carcinoma. An interesting question is whether these different histologic variants or variations are clonally related to conventional acinar carcinoma, or if they may develop independently of the acinar components? Because TMPRSS2:ERG rearrangement is one of the most common genomic events in human prostate carcinoma and constitutes one of the early event in prostate carcinogenesis, ${ }^{26,30}$ we monitored this gene fusion, using it as a tool to study the clonality of the conventional acinar component and histologic variants and determine whether they are related genetically. Notably, $88 \%$ of cases showed concordant TMPRSS2:ERG fusion status between paired histologic variant/ histologic variation and conventional acinar prostate carcinoma foci, suggesting histologic variants are clonally related to the conventional acinar prostate carcinoma. One could hypothesize that both histologic components might be derived from an undifferentiated cell with the capacity for multidirectional differentiation. Alternatively, the variant histologic counterpart could develop from the conventional acinar prostate carcinoma. Of note, when variant histology was seen along with conventional acinar carcinoma as part of the focal or same tumor nodule, both tumor components shared the same TMPRSS2:ETS status. ${ }^{26,31}$ In comparison, when discordance of TMPRSS2:ERG rearrangement 
between paired histologic variant and conventional acinar prostate carcinoma was observed (six cases), it was because they represented separate tumor nodules of multifocal prostate carcinoma. Our group and others have demonstrated that, in the setting of multifocal carcinoma, separate tumor nodules are frequently heterogeneous for TMPRSS2 gene rearrangement indicating independent clonal origin. ${ }^{26,31}$ Therefore, our overall observations suggest that, in the vast majority of cases, variant morphology are clonally related to conventional prostate carcinoma. In a small proportion of multifocal prostate carcinoma cases, however, variant morphology may represent an independent disease.

In this study, $45 \%$ of prostate carcinoma cases with variant morphology did not harbor any ETS aberration. Using a COPA strategy, we have recently identified SPINK1 overexpression in a subset of ETS fusion-negative prostate carcinoma $(\sim 11 \%$ of total cases). ${ }^{21}$ In this cohort, SPINK1 overexpression was identified in $6 \%$ of all ETS negative cases. This frequency is somewhat less but comparable to that reported in conventional acinar prostate carcinoma. ${ }^{21}$ A previous study has found SPINK1 overexpression as an unfavorable clinical parameter in prostate carcinoma. ${ }^{21}$ Interestingly, SPINK1 overexpression was exclusively found in ducal adenocarcinomas in this cohort, which may explain the poor prognosis usually observed in ductal adenocarcinomas of prostate.

In summary, we have suggested a potential molecular connection between the ETS gene fusions and certain histologic variants of prostate carcinoma, most notably, mucinous carcinoma. Exclusive association of TMPRSS2:ERG through Edel mechanism in small cell carcinomas further suggests that TMPRSS2:ERG, through Edel, represents an aggressive molecular subtype of prostate carcinoma. In addition, for the first time, we demonstrate that variant morphology is clonally related to conventional acinar carcinoma, and potentially could represent a tumor clonal expansion of conventional acinar carcinoma. Our data may provide a better understanding of the origin and phenotypic association of ETS fusions in histologic variants of prostate carcinoma, and are potentially implacable in clinical management of these patients with prostate carcinoma.

\section{Acknowledgements}

We thank Anjana Menon for technical assistance and Jill Granger for editorial review of this article. This study was supported in part by Department of Defense (PC040517, W81XWH-06-1-0224), the National Institutes of Health (Prostate SPORE P50CA69568, RO1 CA102872), and the Early Detection Research Network (UO1 CA111275-01). AMC was supported by a Clinical Translational Research Award from the Burroughs Welcome Foundation and an award by the Doris Duke Charitable Foundation. SAT is a fellow of the Medical Scientist Training Program and is supported by the GPC Biotech Young Investigator Award from the Prostate Cancer Foundation.

\section{Disclosure}

The University of Michigan has filed a patent on ETS gene rearrangements in prostate cancer, on which RM, SAT, and AMC are coinventors, and the diagnostic field of use has been licensed to GenProbe Inc. Gen-Probe Inc. has not played a role in the design and conduct of the study nor in the collection, analysis, or interpretation of the data, and no involvement in the preparation, review, or approval of the article. AMC serves as a consultant to Gen-Probe Inc.

\section{References}

1 Jemal A, Siegel R, Ward E, et al. Cancer statistics 2008. CA Cancer J Clin 2008;58:71-96.

2 Che M, Grignon D. Pathology of prostate cancer. Cancer Metastasis Rev 2002;21:381-395.

3 Randolph TL, Amin MB, Ro JY, et al. Histologic variants of adenocarcinoma and other carcinomas of prostate: pathologic criteria and clinical significance. Mod Pathol 1997;10:612-619.

4 Grignon DJ. Unusual subtypes of prostate cancer. Mod Pathol 2004;17:316-327.

5 Tomlins SA, Rhodes DR, Perner S, et al. Recurrent fusion of TMPRSS2 and ETS transcription factor genes in prostate cancer. Science 2005;310:644-648.

6 Tomlins SA, Mehra R, Rhodes DR, et al. TMPRSS2:ETV4 gene fusions define a third molecular subtype of prostate cancer. Cancer Res 2006;66: 3396-3400.

7 Helgeson BE, Tomlins SA, Shah N, et al. Characterization of TMPRSS2:ETV5 and SLC45A3:ETV5 gene fusions in prostate cancer. Cancer Res 2008;68:73-80.

8 Tomlins SA, Laxman B, Dhanasekaran SM, et al. Distinct classes of chromosomal rearrangements create oncogenic ETS gene fusions in prostate cancer. Nature 2007;448:595-599.

9 Han B, Mehra R, Dhanasekaran SM, et al. A fluorescence in situ hybridization screen for E26 transformation-specific aberrations: identification of DDX5-ETV4 fusion protein in prostate cancer. Cancer Res 2008;68:7629-7637.

10 Hermans KG, Bressers AA, van der Korput HA, et al. Two unique novel prostate-specific and androgenregulated fusion partners of ETV4 in prostate cancer. Cancer Res 2008;68:3094-3098.

11 Mehra R, Tomlins SA, Shen R, et al. Comprehensive assessment of TMPRSS2 and ETS family gene aberrations in clinically localized prostate cancer. Mod Pathol 2007;20:538-544.

12 Mehra R, Tomlins SA, Yu J, et al. Characterization of TMPRSS2-ETS gene aberrations in androgen-independent metastatic prostate cancer. Cancer Res 2008;68: 3584-3590. 
$13 \mathrm{Tu}$ JJ, Rohan S, Kao J, et al. Gene fusions between TMPRSS2 and ETS family genes in prostate cancer: frequency and transcript variant analysis by RT-PCR and FISH on paraffin-embedded tissues. Mod Pathol 2007;20:921-928.

14 Rajput AB, Miller MA, De Luca A, et al. Frequency of the TMPRSS2:ERG gene fusion is increased in moderate to poorly differentiated prostate cancers. J Clin Pathol 2007;60:1238-1243.

15 Perner S, Demichelis F, Beroukhim R, et al. TMPRSS2:ERG fusion-associated deletions provide insight into the heterogeneity of prostate cancer. Cancer Res 2006;66:8337-8341.

16 Attard G, Clark J, Ambroisine L, et al. Duplication of the fusion of TMPRSS2 to ERG sequences identifies fatal human prostate cancer. Oncogene 2008;27: 253-263.

17 Demichelis F, Fal K, Perner S, et al. TMPRSS2:ERG gene fusion associated with lethal prostate cancer in a watchful waiting cohort. Oncogene 2007;26: 4596-4599.

18 Nam RK, Sugar L, Yang W, et al. Expression of the TMPRSS2:ERG fusion gene predicts cancer recurrence after surgery for localised prostate cancer. Br J Cancer 2007;97:1690-1695.

19 Tomlins SA, Mehra R, Rhodes DR, et al. Integrative molecular concept modeling of prostate cancer progression. Nat Genet 2007;39:41-51.

20 Iljin K, Wolf M, Edgren H, et al. TMPRSS2 fusions with oncogenic ETS factors in prostate cancer involve unbalanced genomic rearrangements and are associated with HDAC1 and epigenetic reprogramming. Cancer Res 2006;66:10242-10246.

21 Tomlins SA, Rhodes DR, Yu J, et al. The role of SPINK1 in ETS rearrangement-negative prostate cancers. Cancer Cell 2008;13:519-528.

22 Melo JV. The diversity of BCR-ABL fusion proteins and their relationship to leukemia phenotype. Blood 1996;88:2375-2384.
23 Halvarsson B, Muller W, Planck M, et al Phenotypic heterogeneity in hereditary non-polyposis colorectal cancer: identical germline mutations associated with variable tumour morphology and immunohistochemical expression. J Clin Pathol 2007;60: 781-786.

24 Lakhani SR. The pathology of familial breast cancer: morphological aspects. Breast Cancer Res 1999;1: 31-35.

25 Mosquera JM, Perner S, Demichelis F, et al. Morphological features of TMPRSS2-ERG gene fusion prostate cancer. J Pathol 2007;212:91-101.

26 Mehra R, Han B, Tomlins SA, et al. Heterogeneity of TMPRSS2 gene rearrangements in multifocal prostate adenocarcinoma: molecular evidence for an independent group of diseases. Cancer Res 2007;67: 7991-7995.

27 Han B, Mori I, Wang X, et al. Combined small-cell carcinoma of the stomach: p53 and K-ras gene mutational analysis supports a monoclonal origin of three histological components. Int J Exp Pathol 2005;86:213-218.

28 Makretsov N, He M, Hayes M, et al. A fluorescence in situ hybridization study of ETV6-NTRK3 fusion gene in secretory breast carcinoma. Genes Chromosomes Cancer 2004;40:152-157.

29 Mertz KD, Setlur SR, Dhanasekaran SM, et al. Molecular characterization of TMPRSS2-ERG gene fusion in the NCI-H660 prostate cancer cell line: a new perspective for an old model. Neoplasia 2007;9:200-206.

30 Perner S, Mosquera JM, Demichelis F, et al. TMPRSS2ERG fusion prostate cancer: an early molecular event associated with invasion. Am J Surg Pathol 2007;31:882-888.

31 Barry M, Perner S, Demichelis F, et al. TMPRSS2-ERG fusion heterogeneity in multifocal prostate cancer: clinical and biologic implications. Urology 2007;70: 630-633. 